\title{
Analysis of long non-coding RNA expression profiles in clear cell renal cell carcinoma
}

\author{
FEI YAN YANG ${ }^{1}$, YAN WANG $^{1}$, JIAN GUO WU ${ }^{1}$, SHAO LI SONG ${ }^{2}$, GANG HUANG $^{2}$, \\ WEI MIN XI ${ }^{1}$, LI LING TAN ${ }^{1}$, JIAN WANG ${ }^{1}$ and QING CAO $^{3}$ \\ ${ }^{1}$ Department of Nuclear Medicine, Second Affiliated Hospital, Nanchang University, Nanchang, Jiangxi 330006; \\ ${ }^{2}$ Department of Nuclear Medicine, Ren Ji Hospital, Shanghai Jiao Tong University, School of Medicine, Shanghai 200127; \\ ${ }^{3}$ Jiangxi Key Laboratory of Molecular Medicine, Second Affiliated Hospital, Nanchang University, \\ Nanchang, Jiangxi 330006, P.R. China
}

Received January 29, 2017; Accepted June 26, 2017

DOI: $10.3892 / 01.2017 .6563$

\begin{abstract}
To investigate the expression patterns of long non-coding RNAs (lncRNAs) in clear cell renal cell carcinoma (ccRCC) and in metastatic renal cell carcinoma (RCC), the present study downloaded three human exon arrays available from the public Gene Expression Omnibus. The probes of the human exon arrays were re-annotated and the probes uniquely mapping to lncRNAs were retained at the gene level. Following the analysis of GSE53757 and GSE46699, which contained paired ccRCC cancer and normal adjacent tissue samples, 32 differentially expressed IncRNAs (adjusted $\mathrm{P}<0.01$ ) in ccRCC were identified. Various IncRNAs, including ENSG00000177133, NR_024418, T-cell leukemia/lymphoma 6 (TCL6), growth arrest-specific transcript 5, deleted in lymphocytic leukemia 2, colorectal neoplasia differentially expressed (CRNDE) and MIR155HG, have been reported to be abnormally expressed in cancers. Of these genes, NR_24418 and TCL6 have been reported to be associated with ccRCC. Following analysis of GSE47352, which contained 4 primary metastatic and 5 non-metastatic tumor samples, the 50 top differentially expressed lncRNAs were identified in metastatic ccRCC (Mann-Whitney U test, $\mathrm{P}<0.05)$. Comparison with the ccRCC associated lncRNAs revealed that the IncRNA CRNDE demonstrated an increased
\end{abstract}

Correspondence to: Dr Jian Guo Wu, Department of Nuclear Medicine, Second Affiliated Hospital, Nanchang University, 1 MinDe Road, Nanchang, Jiangxi 330006, P.R. China

E-mail: grantwu@foxmail.com;wujgmd@gmail.com

Abbreviations: lncRNAs, long non-coding RNAs; ccRCC, clear cell renal cell carcinoma; VHL, von Hippel-Lindau gene; PBRM1, polybromo-1; SETD2, SET domain containing 2; KDM5C, lysine (K)-specific demethylase 5C; KDM6A, lysine (K)-specific demethylase 6A; BAP1, BRCA1 associated protein-1

Key words: long non-coding RNA, clear cell renal cell carcinoma, microarray analysis, data mining expression in ccRCC and metastatic ccRCC samples, which suggested that CRNDE is important in the progression of ccRCC. The lncRNA ENSG00000244020 was decreased in ccRCC and metastatic ccRCC, suggesting that silencing of ENSG00000244020 may be important in ccRCC development. Overall, a set of lncRNAs was identified as differentially expressed in ccRCC and metastatic ccRCC, providing potential candidates for the discovery of novel cancer biomarkers and therapeutic targets to improve diagnosis and therapy in RCC.

\section{Introduction}

Each year around 250,000 new cases of kidney cancer (KC) are diagnosed in the world, accounting for about $2 \%$ of all cancers (1). It is one of the highest incidences of cancer in the urinary system tumors. The morbidity and mortality of $\mathrm{KC}$ is continuously growing. The clear cell renal cell carcinoma (ccRCC), the most common subtype of $\mathrm{KC}$, is estimated as $80 \%$ of all patients (2). The comprehensive biological process of the molecular mechanism of ccRCC may involve in stepwise accumulation of genomic instability, epigenetic changes, gene mutations and deviant expression of protein-coding genes and noncodings $(3,4)$.

The genome-wide association researches have found several mutations conferring risk of ccRCC, including von Hippel-Lindau gene $(V H L)$, which codes for a tumor suppressor gene. Various causes, such as mutation, loss of heterozygosity, allelic deletion and promoter hypermethylation, makes $V H L$ inactivation on chromosome $3 \mathrm{p}$ as a high frequent genetic event in ccRCC (5). Carcinogenesis and cancer-associated angiogenesis is well-characterized by the alteration in the pathway of $V H L$ gene/hypoxia inducible factor/vascular endothelial growth factor (6). Moreover, some genes, such as $K D M 5 C$ [lysine (K)-specific demethylase 5C], PBRM1 (polybromo-1), BAP1 (BRCA1 associated protein-1), KDM6A [lysine (K)-specific demethylase 6A] and SETD2 (SET domain containing 2), are involved in chromatin modification in primary human renal cell carcinoma (RCC) and their mutations will profoundly change their chromatin organization and their transcriptional program (7). 
On the other side, the non-coding RNA transcripts also showed significant difference in ccRCC, such as overexpression of miRNA-210 (8) and downregulation of miR-141 and miR-200c (9). miRNA-210 is one of the HIF-1 targets, reported to correlate with poor patient survival (8).

Long non-coding RNAs (lncRNAs), mainly locating within nucleus or cytosolic compartment (10), many identified of which are transcribed by RNA polymerase II, belong to a new class of non-coding RNA transcripts more than 200 nucleotides in length. Based on their location regarding protein-coding genes, LncRNAs are classified into five locus biotypes: Sense, antisense, bidirectional, intronic and intergenic (11). IncRNAs can regulate gene expression by the ways of transcriptional regulation, splicing, imprinting, epigenetic regulation and subcellular transport (12). Therefore, IncRNAs are important in gene expression and chromatin remodeling and exhibit previously unrecognized functions, such as promotion of the occurrence, development, proliferation and differentiation of the malignant disease including cancers.

In this study, some lncRNAs were identified to be differentially expressed in cancer while comparing with normal tissue, providing some potential candidates for discovery of new cancer biomarkers and therapeutic targets to improve diagnosis and therapy in ccRCC.

\section{Materials and methods}

Source of data sets. Three data sets of human exon arrays, GSE53757, GSE46699 and GSE47352, were downloaded from GEO. GSE53757 contained 72 paired ccRCC and normal adjacent tissues (13). Data processing and methodology were as previously described (14). GSE46699 contained 65 paired ccRCC and normal adjacent tissues, including stages 1-3 (15). GSE47352 contained 4 primary metastatic and 5 non-metastatic tumor samples (16).

Probe re-annotation pipeline. The following steps as described by Zhang et al (17) were used to assess the lncRNA expressions in the ccRCC gene expression data that a pipeline uniquely mapped to IncRNAs was used to determine the probe sets from the Affymetrix array. First of all, Affymetrix HG-U133 Plus 2.0 (Affymetrix, Santa Clara, CA, USA) probe set ID was mapped to the NetAffx Annotation Files (HG-U133 Plus 2.0 Annotations, CSV format, release 33, 10/30/12), which the Ensembl gene ID, probe set ID, Refseq transcript ID gene symbol, gene symbol, gene title and other informative items for the specific probe set were included in the annotations. Then, we used the following criteria to filter the probe sets: i) 'NR' (NR indicates non-coding RNA in the Refseq database) was labeled and retained for the probe sets which were only assigned with Refseq IDs; ii) 'antisense', 'sense intronic', 'processed transcripts', 'sense overlapping', 'IncRNA' or 'non sense mediated decay' in Ensembl annotations were annotated and retained for the probe sets which were only assigned with Ensembl gene IDs; and iii) those annotated with Ensembl gene titles above and labeled as 'NR' in Refseq database were retained at the same time for the probe sets with both Ensembl gene IDs and Refseq IDs. Secondly, rRNAs, pseudogenes, microRNAs and other short RNAs including snRNAs, tRNAs and snoRNAs were filtered out and 2,935 Affymetrix probe sets with corresponding annotated lncRNA transcripts were obtained.

Data analysis. After quality control assessment was done, each dataset was normalized by applying a Bioconductor package Robust multi-array average (RMA) through $\mathrm{R}$ 3.0.2. Then the linear models for microarray data (LIMMA), a modified t-test method incorporating the Benjamini-Hochberg multiple hypotheses correction technique, were used to analyze the normalized data. The probe sets of which the adjusted P-value was below 0.01 between two groups were defined as significantly different lncRNAs.

\section{Results}

LncRNA expression profiles on Affymetrix HG-U133 Plus 2.0 arrays. According to the Ensembl and Ref-seq annotations of IncRNAs and the NetAffx annotation of probe sets, 2,935 probe sets corresponding to lncRNA genes were identified on the Affymetrix HG-U133 Plus 2.0 arrays. Among them, we annotated 1,002 probe sets mapping to IncRNA genes by both the RefSeq and Ensemble database; we annotated 500 probe sets only by the RefSeq database; we annotated 1,423 probe sets only by the Ensemble database. Those probe sets with controversial annotations were excluded in the two databases.

LncRNAs differentially expressed in ccRCC. We used $\mathrm{R}$ to process the CEL files for background correction, normalization, and summarizations with RMA algorithm. Comparing to normal tissue by using LIMMA at a threshold of an adjusted P-value <0.01, 85 lncRNAs in GSE53737 (Fig. 1) and 32 lncRNAs in GSE46699 (Fig. 2) were identified to be differentially expressed in ccRCC. Interestingly, the overlapping IncRNAs of both arrays were exactly the differentially expressed lncRNAs in GSE46699, listed in Table I. A total of 18 downregulated and 14 upregulated IncRNAs was found among the 32 differentially expressed lncRNAs. Any official Human Genome Nomenclature Committee symbols were not yet owned to most of these IncRNAs and their functions are still unknown. Some lncRNAs, such as ENSG00000177133 (18), NR_024418 (19), T-cell leukemia/lymphoma 6 (TCL6) (20,21), GAS5 (22-25), DLEU2 (26), colorectal neoplasia differentially expressed (CRNDE) (27), and MIR155HG (28), have been reported to play a pro- or anticancer role in various kind of cancers. Among them, NR_24418 and TCL6 have been reported to be associated with ccRCC $(19,20)$ (Table I).

Independent validation. We analyzed GSE46699 and GSE53737 to independently validate the results (Fig. 3), finding that significant expression changes (adjusted $\mathrm{P}<0.01$ ) at the same direction were presented for the above differentially expressed lncRNAs. A significant concordance shown in Fig. 3 was existed in the distribution of expression differentials between the experiment and the validation data sets, reflecting that the expression patterns of these genes were highly consistent among different sample sets.

Differentially expressed lncRNAs associated with RCC metastasis. To validate the utility of exon array data in combination with clinical annotation to identify cancer 


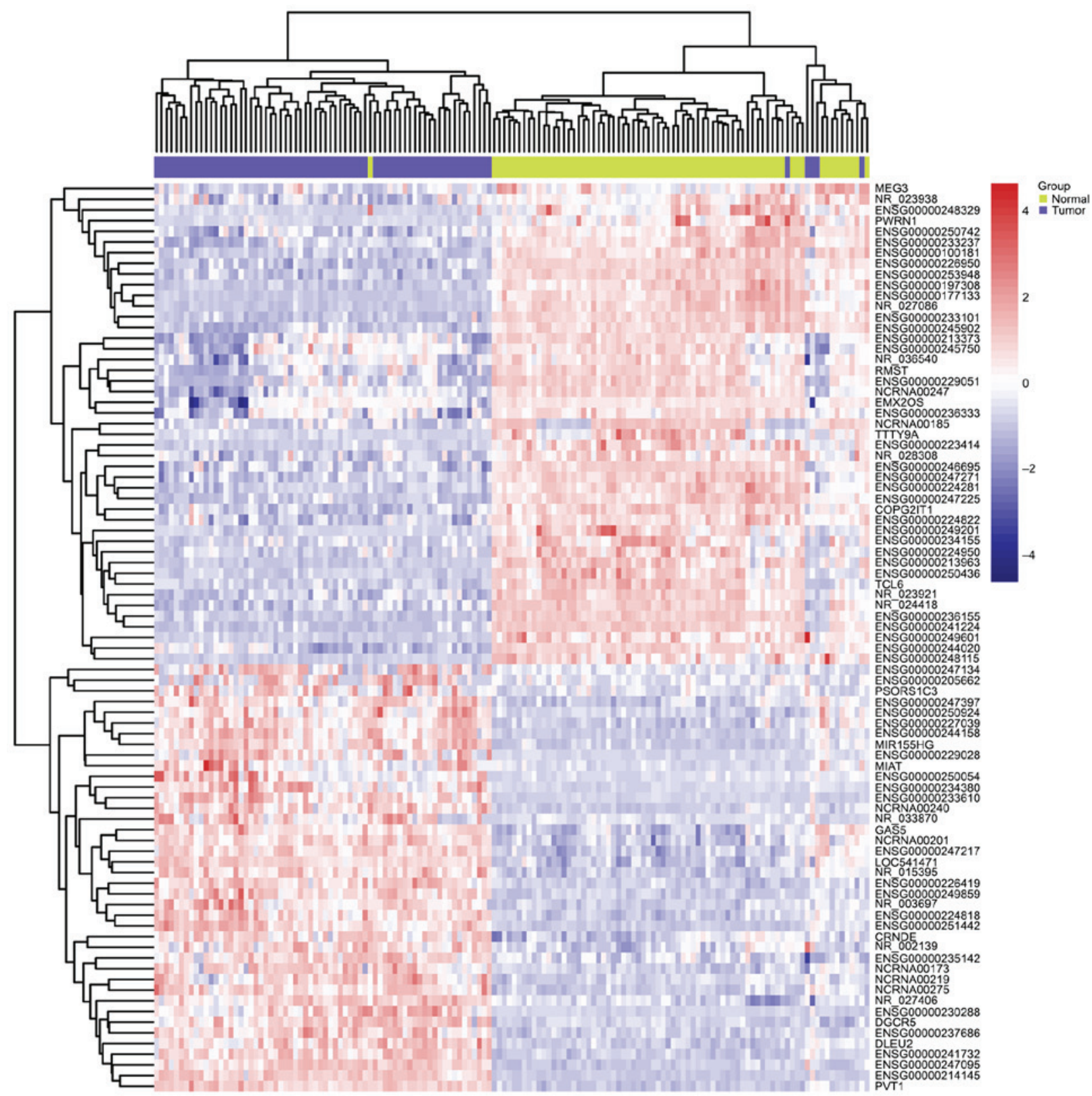

Figure 1. Clustering heatmap of 72 paired normal samples (green) and tumors (blue) based on the 85 differentially expressed lncRNAs in GSE53757. Each column represents one sample and each row represents one lncRNA. Gene expression levels are indicated as follows: Red, high expression; blue, low expression. LncRNAs, long non-coding RNAs; TCL6, T-cell leukemia/lymphoma 6; CRNDE, colorectal neoplasia differentially expressed.

metastasis-related IncRNA, we examined the expression pattern of GSE47352 consisting of 4 primary metastatic and 5 non-metastatic tumor samples (Fig. 4). Comparing to non-metastatic samples, the top 50 differentially expressed IncRNAs in metastatic ccRCC were identified and we found 22 lncRNAs were upregulated and 28 lncRNAs were downregulated (Mann-Whitney $\mathrm{U}$ test, $\mathrm{P}<0.05$ ). By comparing with the results above, we found that three out of these fifty lncRNAs were also present in the overlapping genes shown in Table I, which are ENSG00000214145, CRNDE and ENSG00000244020. Among them, CRNDE showed higher expression in both ccRCC and metastatic samples compared to normal tissue and non-metastatic ones, while ENSG00000244020 is lower expressed. However, the lncRNA ENS00000214145 showed an increased expression in ccRCC but decreased in metastatic RCC.

\section{Discussion}

LncRNAs function in comprehensive biological processes through miscellaneous mechanisms $(29,30)$. In recent years emerging evidence indicate that IncRNAs are disordered and play important roles in tumorigenesis and tumor progression $(10,12,22,27)$. Because the existing microarrays may fortuitously contain some lncRNAs probes, some researchers $(31,32)$ recently suggested that we mine these array data and may achieve lncRNA expression profiling.

In the present study, a set of IncRNAs differentially expressed between ccRCC and normal renal tissue was analyzed. Previous studies have reported some lncRNAs in human cancers, including ENSG00000177133, NR_024418, TCL6, GAS5, DLEU2, CRNDE and MIR155HG, while recent studies have reported NR_24418 and TCL6 associated with ccRCC $(19,20)$. 


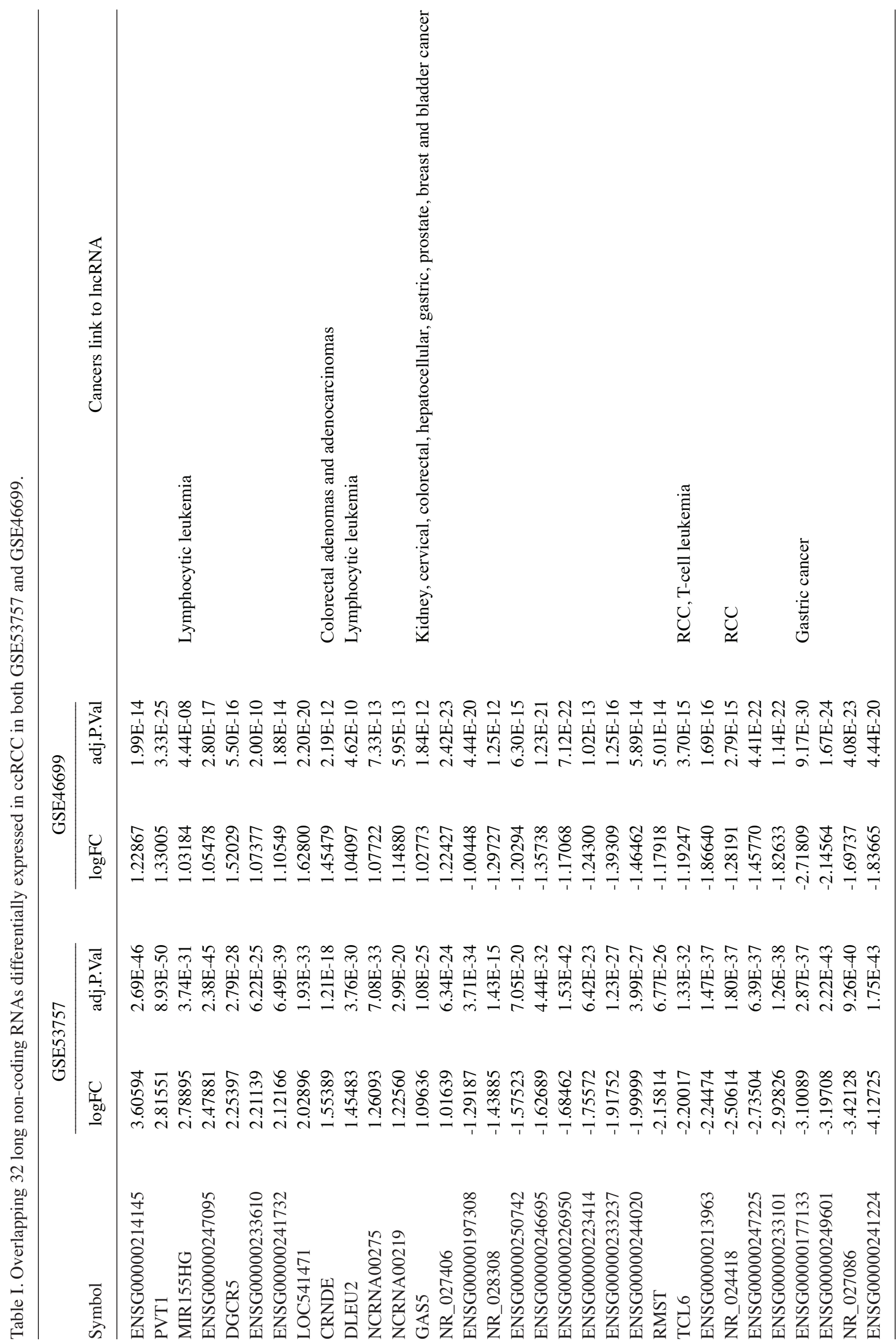




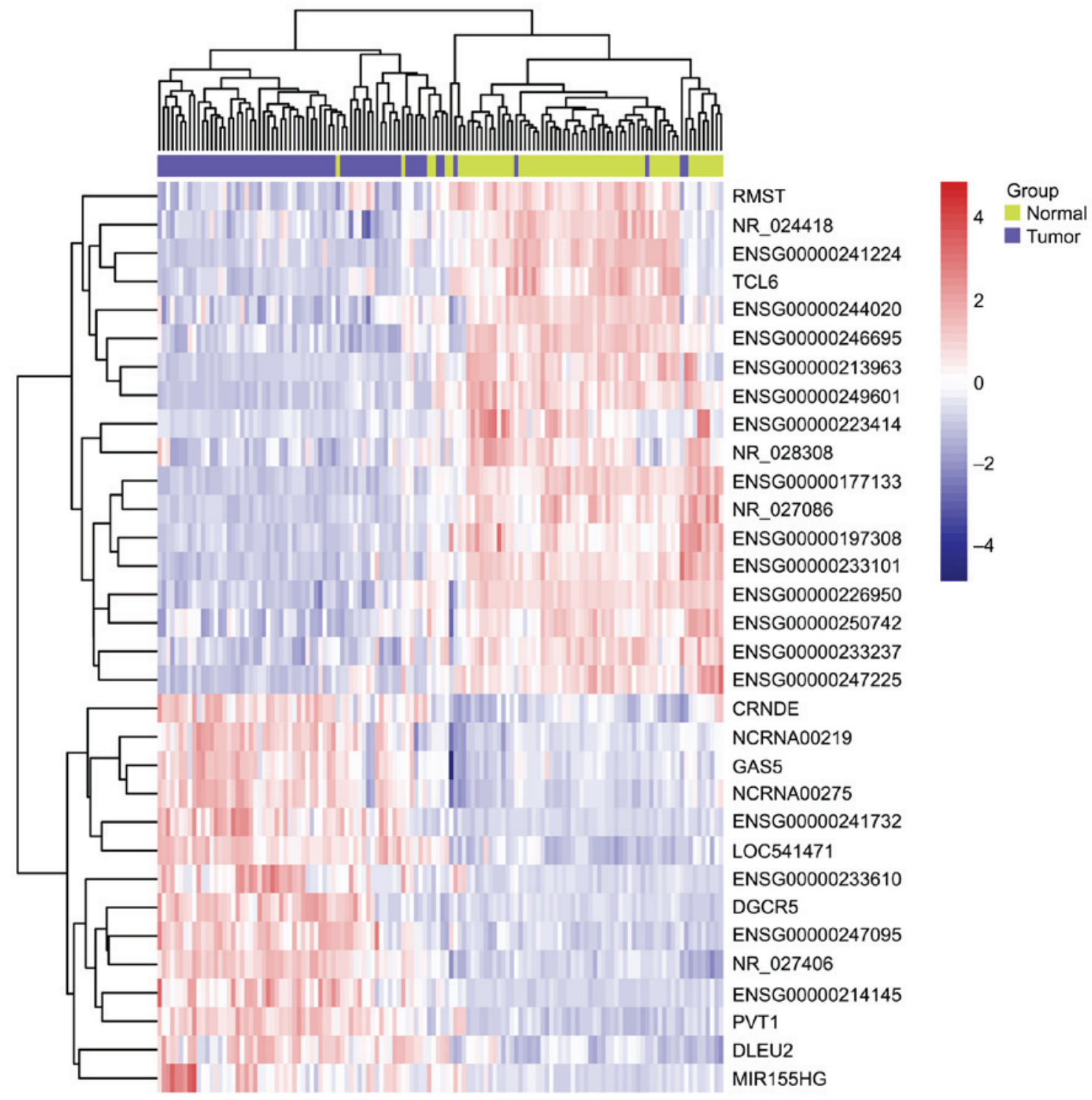

Figure 2. Clustering heatmap of 65 paired normal samples (green) and tumors (blue) based on the 32 differentially expressed lncRNAs in GSE46699. Each column represents one sample and each row represents one lncRNA. Gene expression levels are indicated as follows: red, high expression; blue, low expression. LncRNAs, long non-coding RNAs; TCL6, T-cell leukemia/lymphoma 6; CRNDE, colorectal neoplasia differentially expressed.

TCL6 was first identified in T-cell leukemia (21), located $7 \mathrm{~kb}$ upstream of the TML1 locus on chromosome 14q32.1. The TCL6 gene expressed at least 11 isoforms through very complex alternative-splicing, including splicing with the TML1 gene. Since the TCL6 gene was expressed in T-cell leukemia carrying a $\mathrm{t}(14 ; 14)(\mathrm{q} 11 ; \mathrm{q} 32.1)$ chromosome translocation and was not expressed in normal T-cells, it is thought to be a candidate gene potentially involved in leukemogenesis. Recently, a microarray experiment performed with custom-designed arrays enriched with probes for lncRNAs mapping to intronic genomic regions (20), analyzing 18 primary RCC tumors and 11 non-tumor adjacent matched tissues. Forty lncRNAs differentially expressed between RCC and non-tumor samples was obtained (false discovery rate $<5 \%$ ), including TCL6. However, the fold change (FC) of TCL6 in RCC showed an increased expression $(\mathrm{FC}=1.96)$, which is opposite to our results $(\log F C=-2.20$ in GSE53757 and $\log F C=-1.19$ in GSE46699, $\mathrm{P}<0.01)$. As our study have integrated two microarray analysis, we stand more about our results and it is also

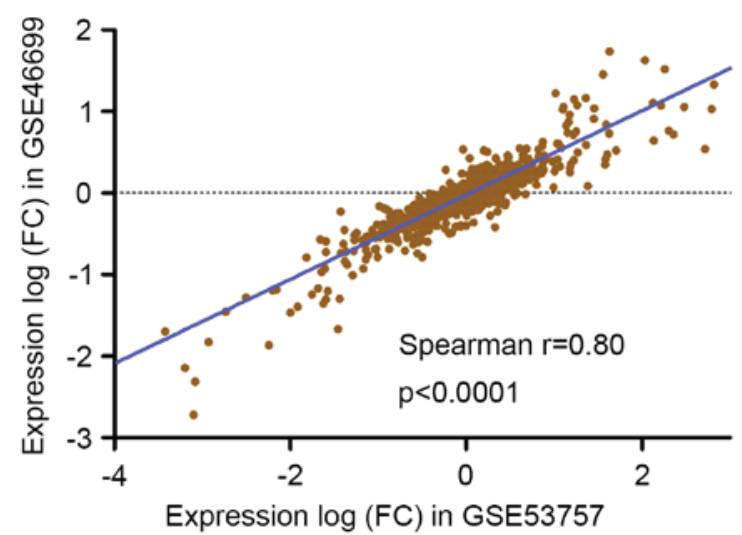

Figure 3. Distribution of expression differentials between experimental data set GSE53757 and validation data set GSE46699. FC, fold change.

necessary to process more research work to find out the role of TCL6 in RCC. 


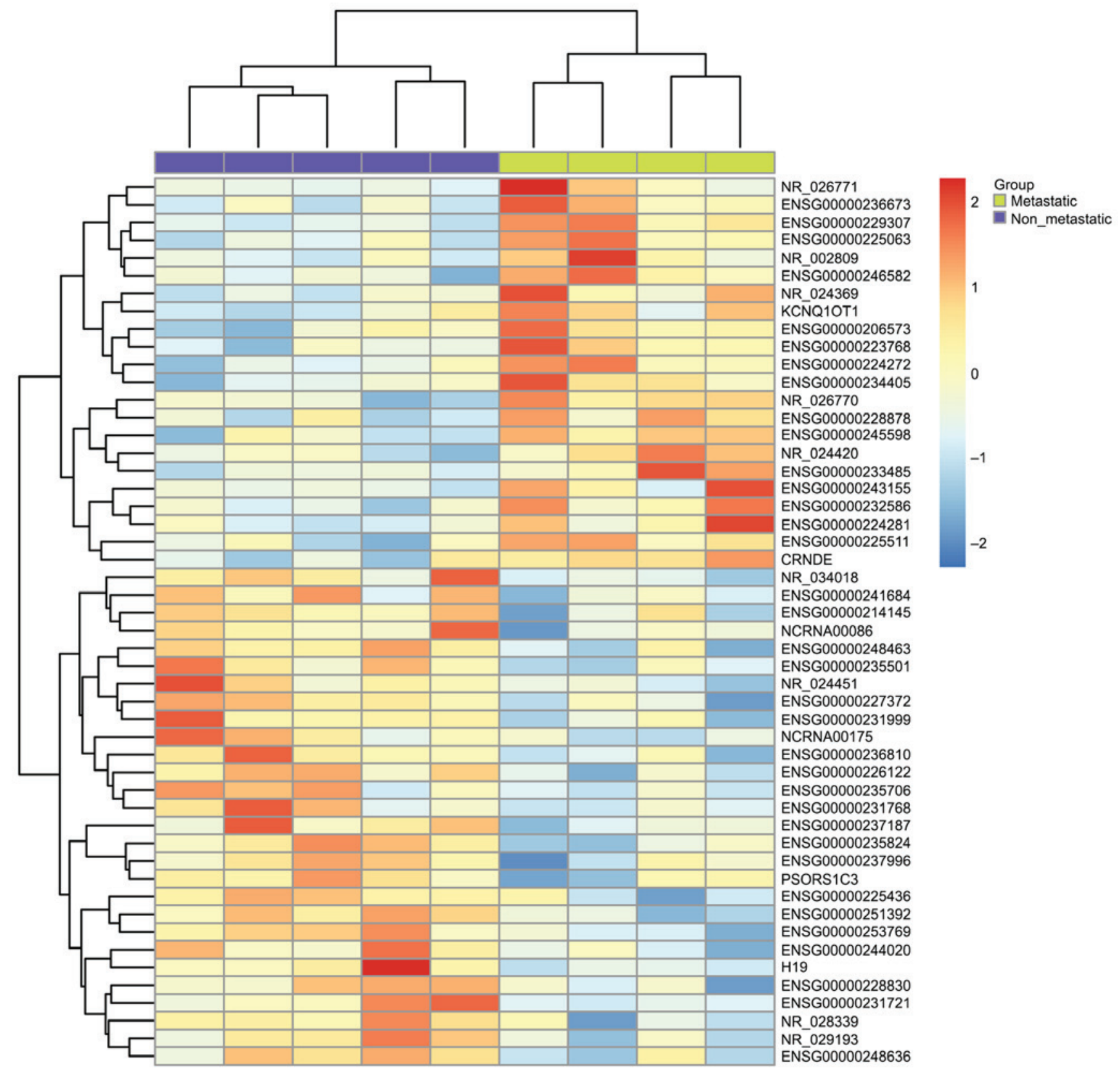

Figure 4. Clustering heatmap of 4 primary metastatic (green) and 5 non-metastatic tumor (blue) samples based on the 50 differentially expressed lncRNAs in GSE47352. Gene expression levels are indicated as follows: Red, high expression; blue, low expression. LncRNAs, long non-coding RNAs; CRNDE, colorectal neoplasia differentially expressed.

Another lncRNA gene, NR_024418, is a 723 bp intragenic lncRNA transcribed from chromosome 5. We found NR_024418 is decreased in ccRCC in two arrays $(\log F C=-2.51$ in GSE53757 and $\log \mathrm{FC}=-1.28$ in GSE46699, $\mathrm{P}<0.01$ ), which is consistent with the results $(\log \mathrm{FC}=-4.16)$ published in 2012 (19). Nevertheless, the characters and biological function of NR_024418 were still unclear.

GAS5 was reported as tumor suppressor in various cancers, including KC (33), colorectal cancer (22), breast cancer (34) and hepatocellular carcinoma (23). RCC tissue and cell line a498 displayed reduced GAS5 expression. Progressed cell death, arrested cell cycle, retarded growth and devoid of invasion and migration were displayed in the cells a498 ectopically expressing GAS5 (35). These findings supported the tumor suppressor role of GAS5 in RCC. GAS5 is critical to control cell apoptosis and cell population growth in mammalian (34). GAS5 transcripts are subject to complex post-transcriptional processing and some, but not all, GAS5 transcripts sensitize mammalian cells to apoptosis inducers. Molecular study identified the GAS5 gene as a novel partner of BCL6 (encoding a $\mathrm{POZ} /$ zinc finger protein that acts as a transcriptional repressor), which is frequently altered at its 5 'non-coding region by $3 q 27$ chromosomal translocation in B-cell lymphoma (36). However, in our study, we found GAS5 upregulated in ccRCC in both arrays $(\log F C=1.10$ in GSE53757 and $\operatorname{logFC}=1.03$ in GSE46699, $\mathrm{P}<0.01$ ). More research work is needed to find out the role of GAS5 in ccRCC.

By comparing the overlapping genes in ccRCC and metastatic ccRCC, we found that the gene CRNDE was increased in both ccRCC samples compared to normal tissues and in 
metastatic RCC compared to non-metastatic RCC, which remind us that CRNDE probably display a crucial role in ccRCC development. CRNDE is transcribed from chromosome 16 on the strand opposite to the adjacent IRX5 gene, with which it may share a bidirectional promoter (37). CRNDE was initially identified highly elevated in colorectal cancer (27), but it is also upregulated in many other solid tumors, such as hepatocellular carcinoma (38), prostate cancer (39), KC (40,41) and hematological malignancies $(42,43)$. CRNDE is associated with a 'stemness' signature, because CRNDE was implicated as one of several lncRNAs required for the maintenance of pluripotency in mouse embryonic stem cells, and thus, potentially involved in tumorigenesis (44). Specifically, at the locus for mouse CRNDE, the promoter was bound by select pluripotency-related transcription factors, including c-Myc and n-Myc, while knockdown of CRNDE was associated with decreased levels of a different suite of critical pluripotency markers: Sox2, Klf4, Nanog, and Oct4. Therefore, we believe that CRNDE is an important gene in ccRCC progression, and more research work was demanded to declare the biological mechanisms in ccRCC.

However, studies about the other genes, such as ENSG00000177133, DLEU2, MIR155HG, ENSG00000214145 and ENSG00000244020 are really rare. The only research that mentioned ENS00000177133 is that it is low expressed in gastric cancer (18). The genes DLEU2 and MIR155HG have been reported to be related to myeloproliferative disorders and leukemia $(26,28,45)$. One research published recently shows that the gene ENSG00000244020 is decreased in pancreatic ductal adenocarcinoma (46). Unfortunately, no report was published about the gene ENSG00000214145 in any other lncRNA study.

We mined three existing microarray data sets and achieved global lncRNA expression profiles in ccRCC. A set of lncRNAs differentially expressed in metastatic RCC were also identified, providing potential candidates for discovery of new cancer biomarkers and therapeutic targets to improve diagnosis and therapy in RCC.

\section{Acknowledgements}

The present study was funded by JiangXi, Provincial Department of Science and Technology (grant no. 20161BBG70202), Jiangxi Provincial Health Commission (grant no. 20161071) and the Second Affiliated Hospital of Nanchang University (grant no. 2014YNLC12014).

\section{References}

1. Siegel R, Ma J, Zou Z and Jemal A: Cancer statistics, 2014. CA Cancer J Clin 64: 9-29, 2014.

2. Patard JJ, Leray E, Rioux-Leclercq N, Cindolo L, Ficarra V, Zisman A, De La Taille A, Tostain J, Artibani W, Abbou CC, et al: Prognostic value of histologic subtypes in renal cell carcinoma: A multicenter experience. J Clin Oncol 23: 2763-2771, 2005.

3. Maher ER: Genomics and epigenomics of renal cell carcinoma. Semin Cancer Biol 23: 10-17, 2013.

4. Rydzanicz M, Wrzesinski T, Bluyssen HA and Wesoły J: Genomics and epigenomics of clear cell renal cell carcinoma: Recent developments and potential applications. Cancer Lett 341: 111-126, 2013.

5. Jonasch E, Futreal PA, Davis IJ, Bailey ST, Kim WY, Brugarolas J, Giaccia AJ, Kurban G, Pause A, Frydman J, et al: State of the science: An update on renal cell carcinoma. Mol Cancer Res 10: 859-880, 2012.
6. Shen C and Kaelin WG Jr: The VHL/HIF axis in clear cell renal carcinoma. Semin Cancer Biol 23: 18-25, 2013.

7. Simon JM, Hacker KE, Singh D, Brannon AR, Parker JS, Weiser M, Ho TH, Kuan PF, Jonasch E, Furey TS, et al: Variation in chromatin accessibility in human kidney cancer links H3K36 methyltransferase loss with widespread RNA processing defects. Genome Res 24: 241-250, 2014.

8. Nakada C, Tsukamoto Y, Matsuura K, Nguyen TL, Hijiya N, Uchida T, Sato F, Mimata H, Seto M and Moriyama M: Overexpression of miR-210, a downstream target of HIF1 $\alpha$, causes centrosome amplification in renal carcinoma cells. J Pathol 224: 280-288, 2011.

9. Nakada C, Matsuura K, Tsukamoto Y, Tanigawa M, Yoshimoto T, Narimatsu T, Nguyen LT, Hijiya N, Uchida T, Sato F, et al: Genome-wide microRNA expression profiling in renal cell carcinoma: Significant down-regulation of miR-141 and miR-200c. J Pathol 216: 418-427, 2008.

10. Ponting CP, Oliver PL and Reik W: Evolution and functions of long noncoding RNAs. Cell 136: 629-641, 2009.

11. Derrien T, Johnson R, Bussotti G, Tanzer A, Djebali S, Tilgner H, Guernec G, Martin D, Merkel A, Knowles DG, et al: The GENCODE v7 catalog of human long noncoding RNAs: Analysis of their gene structure, evolution, and expression. Genome Res 22: 1775-1789, 2012.

12. Enfield KS, Pikor LA, Martinez VD and Lam WL: Mechanistic roles of noncoding RNAs in lung cancer biology and their clinical implications. Genet Res Int 2012: 737416, 2012.

13. von Roemeling CA, Radisky DC, Marlow LA, Cooper SJ, Grebe SK, Anastasiadis PZ, Tun HW and Copland JA: Neuronal pentraxin 2 supports clear cell renal cell carcinoma by activating the AMPA-selective glutamate receptor-4. Cancer Res 74: 4796-4810, 2014

14. Tun HW, Marlow LA, von Roemeling CA, Cooper SJ, Kreinest P, Wu K, Luxon BA, Sinha M, Anastasiadis PZ and Copland JA: Pathway signature and cellular differentiation in clear cell renal cell carcinoma. PLoS One 5: e10696, 2010.

15. Eckel-Passow JE, Serie DJ, Bot BM, Joseph RW, Cheville JC and Parker AS: ANKS1B is a smoking-related molecular alteration in clear cell renal cell carcinoma. BMC Urol 14: 14, 2014.

16. Ni D, Ma X, Li HZ, Gao Y, Li XT, Zhang Y, Ai Q, Zhang P, Song EL, Huang QB, et al: Downregulation of FOXO3a promotes tumor metastasis and is associated with metastasis-free survival of patients with clear cell renal cell carcinoma. Clin Cancer Res 20: 1779-1790, 2014.

17. Zhang X, Sun S, Pu JK, Tsang AC, Lee D, Man VO, Lui WM, Wong ST and Leung GK: Long non-coding RNA expression profiles predict clinical phenotypes in glioma. Neurobiol Dis 48: $1-8,2012$

18. Cao WJ, Wu HL, He BS, Zhang YS and Zhang ZY: Analysis of long non-coding RNA expression profiles in gastric cancer. World J Gastroenterol 19: 3658-3664, 2013.

19. Yu G, Yao W, Wang J, Ma X, Xiao W, Li H, Xia D, Yang Y, Deng K, Xiao H, et al: LncRNAs expression signatures of renal clear cell carcinoma revealed by microarray. PLoS One 7: e42377, 2012.

20. Fachel AA, Tahira AC, Vilella-Arias SA, Maracaja-Coutinho V, Gimba ER, Vignal GM, Campos FS, Reis EM and VerjovskiAlmeida S: Expression analysis and in silico characterization of intronic long noncoding RNAs in renal cell carcinoma: Emerging functional associations. Mol Cancer 12: 140, 2013.

21. Saitou M, Sugimoto J, Hatakeyama T, Russo G and Isobe M: Identification of the TCL6 genes within the breakpoint cluster region on chromosome $14 \mathrm{q} 32$ in T-cell leukemia. Oncogene 19: 2796-2802, 2000.

22. Yin D, He X, Zhang E, Kong R, De W and Zhang Z: Long noncoding RNA GAS5 affects cell proliferation and predicts a poor prognosis in patients with colorectal cancer. Med Oncol 31: $253,2014$.

23. Tu ZQ, Li RJ, Mei JZ and Li XH: Down-regulation of long non-coding RNA GAS5 is associated with the prognosis of hepatocellular carcinoma. Int J Clin Exp Pathol 7: 4303-4309, 2014.

24. Pickard MR and Williams GT: Regulation of apoptosis by long non-coding RNA GAS5 in breast cancer cells: Implications for chemotherapy. Breast Cancer Res Treat 145: 359-370, 2014.

25. Cao S, Liu W, Li F, Zhao W and Qin C: Decreased expression of lncRNA GAS5 predicts a poor prognosis in cervical cancer. Int J Clin Exp Pathol 7: 6776-6783, 2014.

26. Morenos L, Chatterton Z, Ng JL, Halemba MS, ParkinsonBates M, Mechinaud F, Elwood N, Saffery R and Wong NC: Hypermethylation and down-regulation of DLEU2 in paediatric acute myeloid leukaemia independent of embedded tumour suppressor miR-15a/16-1. Mol Cancer 13: 123, 2014. 
27. Graham LD, Pedersen SK, Brown GS, Ho T, Kassir Z, Moynihan AT, Vizgoft EK, Dunne R, Pimlott L, Young GP, et al: Colorectal neoplasia differentially expressed (CRNDE), a novel gene with elevated expression in colorectal adenomas and adenocarcinomas. Genes Cancer 2: 829-840, 2011.

28. Cui B, Chen L, Zhang S, Mraz M, Fecteau JF, Yu J, Ghia EM, Zhang L, Bao L, Rassenti LZ, et al: MicroRNA-155 influences $\mathrm{B}$-cell receptor signaling and associates with aggressive disease in chronic lymphocytic leukemia. Blood 124: 546-554, 2014.

29. Guttman M and Rinn JL: Modular regulatory principles of large non-coding RNAs. Nature 482: 339-346, 2012.

30. Nagano T and Fraser P: No-nonsense functions for long noncoding RNAs. Cell 145: 178-181, 2011.

31. Michelhaugh SK, Lipovich L, Blythe J, Jia H, Kapatos G and Bannon MJ: Mining Affymetrix microarray data for long non-coding RNAs: Altered expression in the nucleus accumbens of heroin abusers. J Neurochem 116: 459-466, 2011.

32. Liao Q, Liu C, Yuan X, Kang S, Miao R, Xiao H, Zhao G, Luo H, $\mathrm{Bu} \mathrm{D}$, Zhao $\mathrm{H}$, et al: Large-scale prediction of long non-coding RNA functions in a coding-non-coding gene co-expression network. Nucleic Acids Res 39: 3864-3878, 2011.

33. Zhou S, Wang J and Zhang Z: An emerging understanding of long noncoding RNAs in kidney cancer. J Cancer Res Clin Oncol 140: 1989-1995, 2014.

34. Mourtada-Maarabouni M, Pickard MR, Hedge VL, Farzaneh F and Williams GT: GAS5, a non-protein-coding RNA, controls apoptosis and is downregulated in breast cancer. Oncogene 28: 195-208, 2009.

35. Qiao HP, Gao WS, Huo JX and Yang ZS: Long non-coding RNA GAS5 functions as a tumor suppressor in renal cell carcinoma. Asian Pac J Cancer Prev 14: 1077-1082, 2013.

36. Ohno H: Pathogenetic role of BCL6 translocation in B-cell non-Hodgkin's lymphoma. Histol Histopathol 19: 637-650, 2004.

37. Cabili MN, Trapnell C, Goff L, Koziol M, Tazon-Vega B, Regev A and Rinn JL: Integrative annotation of human large intergenic noncoding RNAs reveals global properties and specific subclasses. Genes Dev 25: 1915-1927, 2011.

38. Chang Q, Chen J, Beezhold KJ, Castranova V, Shi X and Chen F: JNK1 activation predicts the prognostic outcome of the human hepatocellular carcinoma. Mol Cancer 8: 64, 2009.
39. Pressinotti NC, Klocker H, Schäfer G, Luu VD, Ruschhaupt M, Kuner R, Steiner E, Poustka A, Bartsch G and Sültmann H: Differential expression of apoptotic genes PDIA3 and MAP3K5 distinguishes between low- and high-risk prostate cancer. Mol Cancer 8: 130, 2009.

40. Ooi A, Wong JC, Petillo D, Roossien D, Perrier-Trudova V, Whitten D, Min BW, Tan MH, Zhang Z, Yang XJ, et al: An antioxidant response phenotype shared between hereditary and sporadic type 2 papillary renal cell carcinoma. Cancer Cell 20: 511-523, 2011.

41. Cifola I, Spinelli R, Beltrame L, Peano C, Fasoli E, Ferrero S, Bosari S, Signorini S, Rocco F, Perego R, et al: Genome-wide screening of copy number alterations and $\mathrm{LOH}$ events in renal cell carcinomas and integration with gene expression profile. Mol Cancer 7: 6, 2008.

42. Rager JE and Fry RC: The aryl hydrocarbon receptor pathway: A key component of the microRNA-mediated AML signalisome. Int J Environ Res Public Health 9: 1939-1953, 2012.

43. Broyl A, Hose D, Lokhorst H, de Knegt Y, Peeters J, Jauch A, Bertsch U, Buijs A, Stevens-Kroef M, Beverloo HB, et al: Gene expression profiling for molecular classification of multiple myeloma in newly diagnosed patients. Blood 116: 2543-2553, 2010.

44. Guttman M, Donaghey J, Carey BW, Garber M, Grenier JK, Munson G, Young G, Lucas AB, Ach R, Bruhn L, et al: lincRNAs act in the circuitry controlling pluripotency and differentiation. Nature 477: 295-300, 2011.

45. Iosue I, Quaranta R, Masciarelli S, Fontemaggi G, Batassa EM, Bertolami C, Ottone T, Divona M, Salvatori B, Padula F, et al: Argonaute 2 sustains the gene expression program driving human monocytic differentiation of acute myeloid leukemia cells. Cell Death Dis 4: e926, 2013.

46. Fu XL, Liu DJ, Yan TT, Yang JY, Yang MW, Li J, Huo YM, Liu W, Zhang JF, Hong J, et al: Analysis of long non-coding RNA expression profiles in pancreatic ductal adenocarcinoma. Sci Rep 6: 33535, 2016. 